\title{
Caracterización de Propiedades Fisicoquímicas de los Suelos de la Zona Cafetera del Municipio de Isnos con el Fin de Establecer su Aptitud de Uso y Manejo
}

\section{Characterization of Physicochemical Properties of Soils in the Coffee Zone Isnos Municipality in Order to Establish its Fitness for Use and Handling}

\author{
Armando Torrente T. ${ }^{1}$ y Álvaro Ladino P. ${ }^{2}$
}

\begin{abstract}
Resumen
Con el propósito de caracterizar las propiedades de los suelos de la zona cafetera del municipio de Isnos, estableciendo su aptitud de uso y manejo, se tomaron como referentes los perfiles de suelo identificados según clasificación de la Federación Nacional de Cafeteros (1985), comprendiendo las unidades cartográficas del Ecotopo Cafetero 214B: San Agustín (Melanudands); Isnos (Basaltos); El Mortiño (Dystropets); Saladoblanco (Toba Volcánica); Magdalena (Lithic Ustorthent), entre otras. Además se analizaron y recomendaron 300 muestras de suelos aleatorias (2008), en el Laboratorio Multilab Agroanalitica de CENICAFE, para la fertilización del cultivo. Se utilizaron aerofotografías y software Autocad, Surfer y Arcgis para la georeferenciación en un mapa base y así crear mapas de variabilidad espacial por cada parámetro evaluado en los análisis de suelos, que junto al manejo estadístico permitió apreciar la variabilidad físico-química y homogenizar los suelos por unidad. El ecotopo corresponde principalmente a entisoles e inceptisoles, con $\mathrm{pH}$ ácidos adecuados al cultivo de café, altos contenidos de materia orgánica, texturas moderadamente pesadas, deficientes en fósforo, sin mayores restricciones en los demás elementos. En dos sectores se presentaron altos valores de aluminio, correlacionados con baja concentración de calcio, magnesio y $\mathrm{pH}$. La clasificación agrológica del IGAC, permitió determinar que las unidades de suelos son aptas para la explotación agrícola, excepto la unidad Magdalena. Principalmente se ubican en las clases II y III, exigiendo prácticas moderadas de manejo y conservación de suelos. Esta clasificación se plasmó en un mapa a escala 1: 25000.
\end{abstract}

Palabras Clave: Unidad cartográfica, zona cafetera, clasificación agrológica, análisis de suelos, ecotopo.

\begin{abstract}
For the sake to characterize the properties of the soils of the coffeein the Isnos Municipality, establishing their fitness for use and management, were taken as referring of the profiles of soil identified according to previous classification made by the Federación Nacional de Cafeteros (1985), comprising the cartographic unities of the Coffee Ecotopo 214B: San Agustín (Melanudands); Isnos (Basalts); El Mortiño (Dystropets); Saladoblanco (Volcanic Toba); Magdalena (Lithic Ustorthent), among others. Besides 300 random ground samples were analyzed (2008), in the laboratory Multilab Agroanalitica of CENICAFE, that gave the recommendations for the fertilization of the cultivation. Aerial photographs were used and software Autocad, Surfer and Arcgis for georeferencing in a map base and to create maps of maps of space variability by every parameter evaluated in the analysis of soil, which together with the statistical analysis allowed us to evaluate the behavior physico-chemical, demonstrating greater similarity within the soils of the same unit. The ecotopo mainly corresponds to entisoles and inceptisoles, with acid $\mathrm{pH}$ proper for the culture of coffee, high organic matter contents, textures moderately weighed, deficient in phosphorus, without main problems in the other elements. In two sectors presented high values of aluminum, being correlated to low concentrations of calcium, magnesium and $\mathrm{pH}$. Based on the classification agrologic IGAC, allowed to determine that characterized soil units are suitable for farming, except Magdalena unit. Mainly situate into Class II and III, requiring moderate practices of managing and conservation of soils. This classification was formed into a final map scale 1: 25000 .
\end{abstract}

Keywords: cartographic unit, coffee zone, agrologic classification, analysis of soils, ecotopo.

1 Ingeniero Agrícola. Doctor en Ciencias Agropecuarias. Docente Universidad Surcolombiana. Avenida Pastrana Carrera $1^{a}$. armando.torrente@ gmail.com

2 Ingeniero Agrícola. Universidad Surcolombiana Neiva. Avenida Pastrana Carrera 1ª Ladingeniero@gmail.com 


\section{Introducción}

La productividad de los suelos está determinada por sus características físicas y químicas, que integradas con las climáticas determinan su producción con un sistema específico de cultivo, o sea, la "vocación" de los suelos. Tomando en cuenta previas clasificaciones de suelos del país por parte del Instituto Geográfico Agustín Codazzi IGAC, del Instituto Colombiano de Hidrología, Meteorología y Adecuación de Tierras HIMAT, del Instituto Colombiano Agropecuario ICA, que utilizaron principalmente variables de vocación agrícola, balances hídricos decadales, áreas agroecológicas y físiografía, el Centro Nacional de Investigaciones del Café CENICAFE, subdividió la zona cafetera colombiana en 86 ecotopos cafeteros según el sistema geográfico, variables climáticas, variables del suelo y cartografía (Gómez et al., 1991). El ecotopo del municipio de Isnos es el 214B (FNC, 1985).

Se identificaron para este estudio algunas condiciones físico-químicas de los suelos de la región cafetera del municipio de Isnos en 300 análisis de suelos con el ánimo de caracterizar potenciales sistemas de diversificación agrícola por clases agrológicas y series de suelos, mediante mapas de variabilidad espacial y el análisis estadístico. Los parámetros evaluados fueron: Textura, pH, Materia Orgánica, Fósforo, Potasio, Magnesio, Calcio, Aluminio y porcentaje de Saturación de Aluminio, lo que además sirvió para ajustarse al plan de fertilización de Cenicafé que hizo las recomendaciones para las siembras establecidas.

Las clases agrológicas son ocho, algunas con arreglo a sus posibilidades mediante el uso y manejo que mejor se adapte, la clase VIII se recomienda solo para uso recreativo (Salinas, 2001). Los suelos de la zona cafetera del municipio de Isnos principalmente corresponden a las Clases II y III.

\section{Metodología}

2.1 Localización: El municipio de Isnos está ubicado en el Macizo Colombiano al sur del departamento del Huila. El casco urbano se ubica a $1700 \mathrm{msnm}$., central al área rural cafetera. La figura 1 muestra la ubicación de las unidades cartográficas en la zona cafetera del municipio de Isnos, mientras en la figura 2 se observan los sitios muestreados y georeferenciados; Los mapas se encuentran a una escala aproximada de 1: 350000, con excepción de la figura 1 (1: 150000).
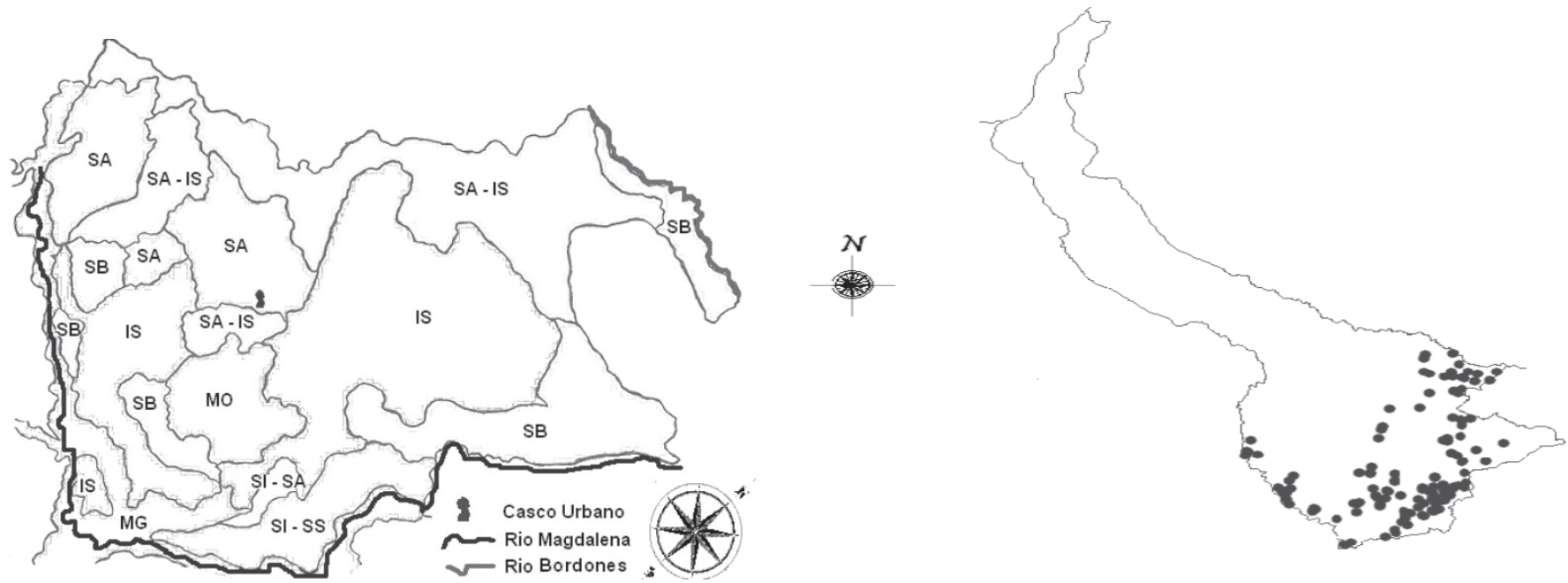

Figura 1. Unidades de suelo de la zona cafetera del municipio de Isnos.

Fuente: Federación Nacional de Cafeteros, 1985

2.2. Extensión: Para alcanzar las metas propuestas por el Comité Departamental de Cafeteros se llegó al agricultor mediante acompañamiento del servicio de extensión rural, realizando actividades como reuniones de capacitación, demostraciones de método y visitas a fincas, promoviendo así la práctica de realizar análisis de suelos, siguiendo las instrucciones según Carrillo et al., 1995. 
2.3 Georeferenciación: Se utilizaron fotografías del IGAC, a escala 1: 10000 y 1: 25000. El área encargada del SICA entregó las coordenadas y luego su georeferenciación. Por último se digitalizó en el programa Arcgis.

2.4 Muestras de suelo: Se tomaron las muestras de suelo por sitio y fueron enviadas al Laboratorio siguiendo los protocolos para tal fin.

2.5 Agrupación de la información: se realizaron los análisis estadísticos respectivos por las unidades de suelo y se identificó el Plan de fertilización.

2.6 Clasificación agrológica: se estimó el uso y manejo de los suelos para finalmente agruparlos según la clasificación agrológica del IGAC.

\section{Resultados}

3.1Métodos de extensión: Se obtuvieron 300 muestras de suelos, una por cada ocho productores, como se aprecia en la tabla 1. Las veredas participantes representan más del 98\% de la caficultura del municipio de Isnos.

Tabla 1. Características del muestreo en relación al estado de la caficultura

\begin{tabular}{|c|c|c|c|c|}
\hline $\begin{array}{c}\text { PANORAMA } \\
\text { CAFETERO }\end{array}$ & VEREDAS & PREDIOS & CAFICULTORES & $\begin{array}{c}\text { ÁREA EN CAFÉ } \\
\text { (Has) }\end{array}$ \\
\hline Municipio de Isnos & 50 & 2521 & 1991 & 2330.13 \\
\hline Zona muestreada & 42 & 254 & 244 & 250 aprox. \\
\hline
\end{tabular}

Fuente: Federación Nacional de Cafeteros de Colombia 2008

3.2. Análisis estadístico: Se aplica muestreo completamente al azar, observando tendencia a mayor homogeneidad por unidad de suelo. Se incluye el promedio de los valores, desviación estándar y coeficiente de variación (tablas 2 y 3). Los datos de mayor variabilidad fueron fósforo y aluminio, en tanto que el pH mostró menor variabilidad.

Tabla 2. Evaluación estadística del Ecotopo 214 B

\begin{tabular}{|c|c|c|c|c|c|c|c|c|c|c|c|}
\hline Parámetros & pH & M.O & P & K & Ca & Mg & Al & Sat. Al & $\mathbf{C a} / \mathbf{M g}$ & $\mathbf{M g} / \mathbf{K}$ & $\mathbf{C a} / \mathbf{k}$ \\
\hline Promedio & 5,3 & 8,7 & 13,9 & 0,4 & 4,6 & 1,8 & 0,5 & 12,1 & 2,7 & 6,3 & 15,8 \\
\hline Desv. Estándar & 0,42 & 4,4 & 23,8 & 0,2 & 2,9 & 1,3 & 0,8 & 18,9 & 1,2 & 5,5 & 12,9 \\
\hline Coefic. Variac. & 0,08 & 0,5 & 1,72 & 0,63 & 0,64 & 0,73 & 1,39 & 1,56 & 0,43 & 0,88 & 0,81 \\
\hline
\end{tabular}

Total: 300 muestras.

Tabla 3. Evaluación estadística de la unidad San Agustín-Isnos

\begin{tabular}{|c|c|c|c|c|c|c|c|c|c|c|c|}
\hline Parámetros & pH & M.O & P & K & Ca & Mg & Al & Sat. Al & $\mathbf{C a} / \mathbf{M g}$ & $\mathbf{M g} / \mathbf{K}$ & $\mathbf{C a} / \mathbf{k}$ \\
\hline Promedio & 5,0 & 11,4 & 6,8 & 0,4 & 2,6 & 1,1 & 1,2 & 31,0 & 2,6 & 3,2 & 8,3 \\
\hline Desv. Estándar & 0,4 & 4,3 & 9,6 & 0,3 & 2,6 & 1,4 & 0,8 & 21,8 & 1,0 & 2,1 & 5,7 \\
\hline Coefic. Variac. & 0,07 & 0,38 & 1,41 & 0,71 & 0,97 & 1,24 & 0,67 & 0,70 & 0,40 & 0,66 & 0,69 \\
\hline
\end{tabular}

Total: 34 muestras.

La textura se determinó por el método organoléptico y su análisis estadístico se basó en las repeticiones de la clase textural por serie cartográfica (tabla 4). 
Tabla 4. Distribución de las texturas por unidad de suelo

\begin{tabular}{|c|c|c|c|c|c|c|c|c|c|}
\hline Textura & SA & IS & SB & MO & MG & SA-IS & SI-SS & SI-SA & Ecotopo \\
\hline FArA & 13 & 20 & 42 & 18 & 13 & 7 & 25 & 12 & 150 \\
\hline FAr & 2 & 14 & 15 & 7 & 4 & 9 & 14 & 3 & 68 \\
\hline FA & 4 & 12 & 10 & 0 & 0 & 13 & 0 & 0 & 39 \\
\hline $\mathrm{Ar}$ & 3 & 12 & 2 & 2 & 2 & 3 & 1 & 2 & 27 \\
\hline F & 2 & 8 & 1 & 0 & 0 & 2 & 1 & 1 & 15 \\
\hline ArA & 0 & 0 & 1 & 0 & 0 & 0 & 0 & 0 & 1 \\
\hline TOTAL & $\mathbf{2 4}$ & $\mathbf{6 6}$ & $\mathbf{7 1}$ & $\mathbf{2 7}$ & $\mathbf{1 9}$ & $\mathbf{3 4}$ & $\mathbf{4 1}$ & $\mathbf{1 8}$ & $\mathbf{3 0 0}$ \\
\hline
\end{tabular}

SA: Unidad San Agustín

IS: Unidad Isnos

SB: Unidad Saladoblanco

MO: Unidad El Mortiño
MG: Unidad Magdalena

SA-IS: Unidad San Agustín-Isnos

SA-IS: Unidad San Agustín-San Simón

SI-SA: Unidad Siberia-San Agustín

3.3. Fertilización: Las recomendaciones fueron dadas para plantaciones con poco sombrío y alta densidad, donde los ajustes los hace el técnico a las dosis (Sadeghian, 2008). De los análisis se identificaron 41 sugerencias para las diferentes etapas del cultivo (crecimiento vegetativo o en producción); principalmente obedecieron a fertilizantes sintéticos y enmiendas de origen natural. Existen algunas restricciones de acuerdo a las concentraciones de los parámetros evaluados, generalmente materia orgánica y bases intercambiables.

En la tabla 5, se aprecia las recomendaciones para la fase vegetativa del café. Se describe el plan de fertilización por tiempos de aplicación, dosis y restricciones, bien sea para fertilización o encalamiento (enmiendas) de forma simple o compuesta, con su grado de fertilizante (Cenicafé, 1994). La fertilización es limitada en cuanto a aplicaciones orgánicas; no se hace mención a fertilización foliar, ni fertirriego.

Tabla 5. Recomendación durante el crecimiento vegetativo en siembra para el cultivo de café (gr/planta)

\begin{tabular}{|c|c|c|c|c|c|c|c|c|c|c|}
\hline \multirow{2}{*}{ 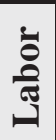 } & \multirow{2}{*}{ Insumo(s) y Fórmula } & \multicolumn{8}{|c|}{ Mes } & \multirow{2}{*}{ Restricción } \\
\hline & & $\mathbf{0}$ & 2 & 6 & 10 & 11 & 14 & 18 & 20 & \\
\hline \multirow{4}{*}{ : } & Úrea & & $15 / 20$ & $20 / 25$ & & & $30 / 35$ & & & \multirow{2}{*}{ M.O>8\% } \\
\hline & $(46 \% \mathrm{~N})$ & & & & & & & & & \\
\hline & Úrea : DAP (3:2) & & & & $25 / 30$ & & & $35 / 40$ & & \multirow{2}{*}{ M.O $<8.0 \%$} \\
\hline & $(35 \% \mathrm{~N}, 18 \% \mathrm{P} 2 \mathrm{O} 5)$ & & & & & & & & & \\
\hline \multirow{4}{*}{ 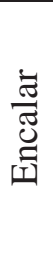 } & Cal Dolomita & 100 & & & & 100 & & & 200 & $\mathrm{Ca}<2,4 \mathrm{me} / 100 \mathrm{~g}$ \\
\hline & $(30 \% \mathrm{Ca} 2 \mathrm{O}, 15 \% \mathrm{MgO})$ & & & & & & & & & $\mathrm{Mg}<0,8 \mathrm{me} / 100 \mathrm{~g}$ \\
\hline & Roca Fosfórica & 40 & & & & 40 & & & 80 & $\mathrm{Ca}<2,4 \mathrm{me} / 100 \mathrm{~g}$ \\
\hline & $\begin{array}{c}(22 \text { a } 30 \% \text { P2O5, } 40 \% \\
\text { Ca) }\end{array}$ & & & & & & & & & $\mathrm{P}<14 \mathrm{ppm}$ \\
\hline
\end{tabular}

Fuente: Sadeghian, 2008

3.4. Variabilidad espacial: Se realizaron mapas que describen la distribución geográfica para cada parámetro evaluado en los análisis de suelos, de acuerdo a los sitios georeferenciados. También se hicieron mapas de variabilidad espacial para las relaciones catiónicas $\mathrm{Ca} / \mathrm{Mg}, \mathrm{Ca} / \mathrm{K}$ y Ca/K. Dos de estos mapas, junto a su respectiva interpretación (Figuras 3 y 4, tablas 6 y 7) se adjuntan a continuación: 


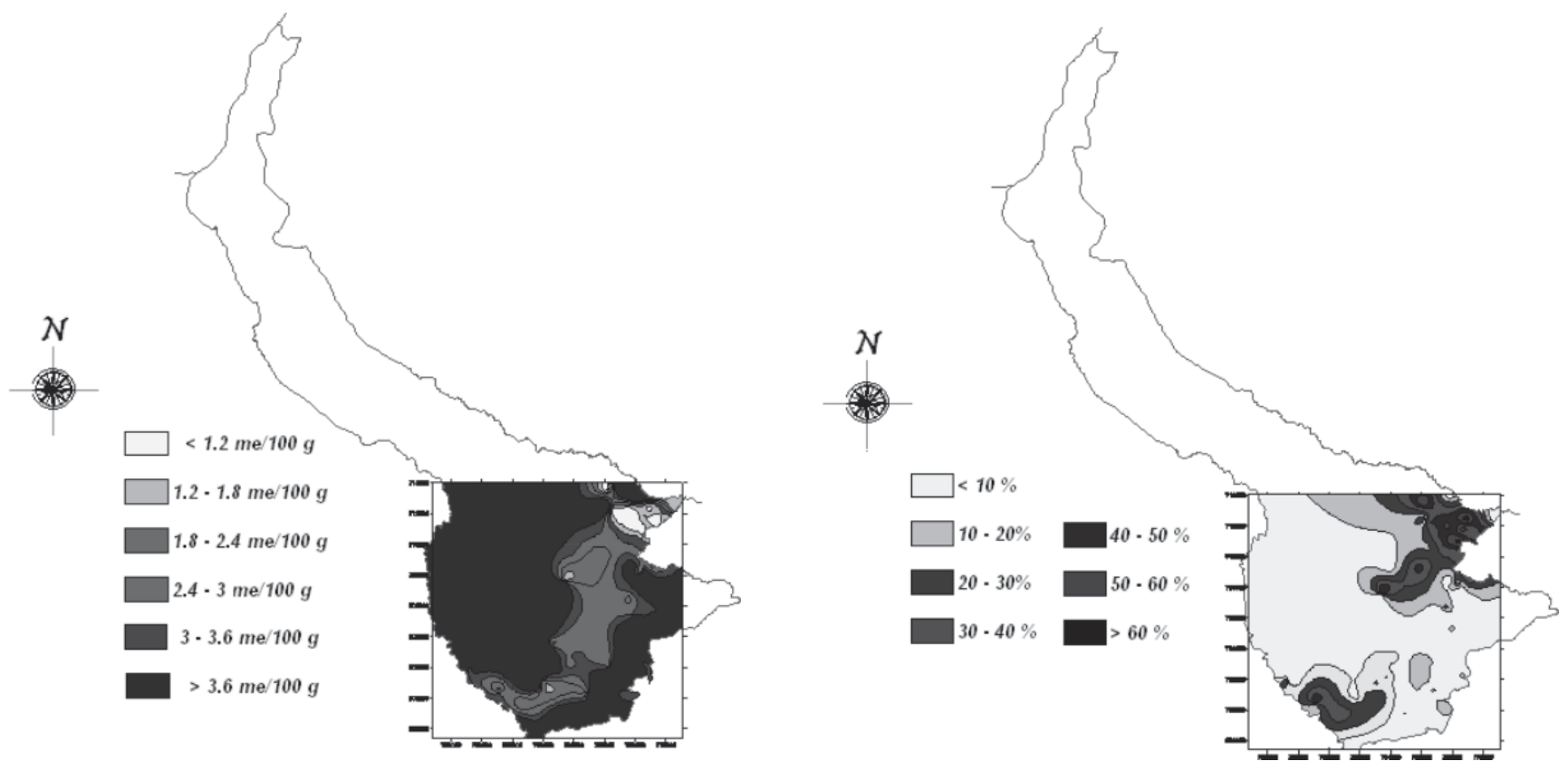

Figura 3. Variabilidad espacial del calcio

Tabla 6. Interpretación del calcio en el suelo

Figura 4. Variabilidad espacial de saturación de aluminio

\begin{tabular}{|c|c|c|c|}
\hline BAJO & MEDIO & ALTO & UNIDAD \\
\hline$<3$ & 3 a 6 & $>6$ & $\mathrm{meq} / 100 \mathrm{~g}$. \\
\hline
\end{tabular}

Tabla 7. Interpretación de la saturación de aluminio en el suelo

Fuente: Sociedad Colombiana de la Ciencia del Suelo, 1990

\begin{tabular}{|c|c|c|c|c|}
\hline $\begin{array}{c}\text { MUY } \\
\text { BAJO }\end{array}$ & BAJO & ALTO & $\begin{array}{c}\text { MUY } \\
\text { ALTO }\end{array}$ & UNIDAD \\
\hline$<15$ & 15 a 30 & 30 a 60 & $>60$ & $\%$ \\
\hline
\end{tabular}

Fuente: Instituto Geográfico Agustín Codazzi

El calcio se presenta en niveles medios a altos en la zona de estudio, la gran mayoría por encima de los 3,6 me/100 g (Figura 3), llegando incluso a valores superiores a 6,0; sin embargo en una franja continua y extendida se reduce la cantidad de este elemento en el suelo.

Los valores mínimos de saturación de aluminio ocurren en las unidades San Agustín y Saladoblanco, mientras los sitios críticos correlacionan con las deficiencias de calcio, magnesio y $\mathrm{pH}$ bajos. En general no se presentan problemas, pero se sugiere aplicar enmiendas según el plan de fertilización. El café es uno de los cultivos más tolerantes a aluminio en el suelo.

3.5. Clasificación agrológica: Se consideraron los siguientes parámetros: permeabilidad, pendiente, profundidad efectiva y grado de erosión, información colectada de la descripción de perfiles de suelo de la zona cafetera de Isnos según clasificación de la Federación Nacional de Cafeteros de Colombia (1985), La información de otros factores como $\mathrm{pH}$, materia orgánica, textura superficial y uso actual de los terrenos fueron tomados de los análisis de las 300 muestras de suelo (2008).

Considerando las particularidades de las clases agrológicas según Salinas (2001), la zona de estudio se clasifica en las clases I, y mayormente II, III, clases aptas para uso agrícola. La unidad Magdalena se ubica entre las clases VI y VII, mientras la unidad Saladoblanco, varía entre clasificación II y III de acuerdo a su ubicación.

\section{Conclusiones}

Los suelos de la zona cafetera del municipio de Isnos en condiciones generales pertenecen a los órdenes taxonómicos Entisoles e Inceptisoles, presentando altos contenidos de materia orgánica, $\mathrm{pH}$ ácido, texturas franco 
arcillosa a franco arcillo arenosa, deficiencias de fósforo y concentraciones medias a altas de los demás elementos químicos evaluados.

Las mayores concentraciones de aluminio en el suelo correlacionan geográficamente con bajos valores de $\mathrm{pH}$, calcio y magnesio. Los suelos de la zona cafetera del municipio de Isnos no superan el $60 \%$ en saturación de aluminio, umbral máximo permitido para los cultivos en general.

El plan de fertilización tradicional basado en los análisis de suelos es muy general, teniendo solo en cuenta los resultados de las propiedades físico-químicas del suelo y la edad del cultivo, pero no incluyen un diagnóstico particular del mismo, ni factores tales como sombrío, densidad, topografía entre otros. Las recomendaciones no dan prelación en marcas comerciales, ni opciones directas en materia de costos de fertilizantes y enmiendas.

Las correctivos de suelos recomendados para la zona cafetera del Ecotopo 214B corresponden a fuentes de calcio y magnesio esencialmente, con suministros de fósforo, tal como la Roca Fosfórica. Las cales fueron Dolomíta y afines en aplicación individual.

Las unidades de suelo de la zona de estudio pertenecen principalmente a Clases Agrológicas II y III. Se deben realizar prácticas de manejo y conservación de suelos para su sostenibilidad.

La variabilidad espacial de las propiedades químicas del suelo permitió conocer la tendencia de los parámetros y componentes mapificados en la zona de estudio.

\section{Referencias Bibliográficas}

1. Carillo, F.I.; S. Suarez, J.R. Sanz, 1995. Como Obtener una Buena Muestra para el Análisis de Suelos. Centro Nacional de Investigaciones de Café. CENICAFÉ. Avance Técnico 214, Chinchiná, Caldas. 4 p.

2. Centro Nacional de Investigaciones de Café, 1994. La Fertilización de los Cafetales Basados en el Análisis de Suelos, la Mejor Inversión. Chinchiná, Caldas. Avance Técnico 202. 8 P.

3. Federación Nacional de Cafeteros de Colombia - FNC, 1985. Estudio de Zonificación y uso Potencial

del Suelo en la Zona Cafetera del Departamento del Huila. Bogotá. 235 p.

4. Gómez G., L. Caballero R., A. y Baldión R., J. V., 1991. Ecotopos Cafeteros. Federación Nacional de Cafeteros de Colombia: Cenicafé - Agroclimatología, División de Desarrollo Social. Bogotá. 125 p.

5. Sadeghian K., S., 2008. Fertilidad del Suelo y Nutrición del Café en Colombia. Centro Nacional de Investigaciones de Café. Chinchiná, Caldas. p.

6. Salinas T., F., 2001. Clasificación Agrológica de los Suelos con Fines Agrícolas. Universidad

Surcolombiana.

Neiva. p. 112.

7. Sociedad Colombiana de la Ciencia del Suelo, 1990. Fundamentos para la Interpretación de Análisis de Suelos, Plantas y Agua para Riego, $3^{\circ}$ Edición. Bogotá. p.157. 